\title{
Two-dimensional solitons in Bose-Einstein condensates with a disk-shaped trap
}

\author{
Guoxiang Huang, ${ }^{1,2}$ Valeri A. Makarov, ${ }^{3}$ and Manuel G. Velarde ${ }^{3,4}$ \\ ${ }^{1}$ Key Laboratory for Optical and Magnetic Resonance Spectroscopy and Department of Physics, East China Normal University, \\ Shanghai 200062, China \\ ${ }^{2}$ The Abdus Salam International Center for Theoretical Physics, P.O. Box 586, Strada Costiera 11, Trieste 34100, Italy \\ ${ }^{3}$ Instituto Pluridisciplinar, Universidad Complutense de Madrid, Paseo Juan XXIII, No. 1, Madrid 28040, Spain \\ ${ }^{4}$ International Center for Mechanical Sciences (CISM), Palazzo del Torso, Piazza Garibaldi, Udine 33100, Italy
}

(Received 4 April 2002; published 13 February 2003)

\begin{abstract}
We consider, both analytically and numerically, the evolution of two-dimensional (2D) nonlinear matterwave pulses in a Bose-Einstein condensate with a disk-shaped trap and repulsive atom-atom interactions. Due to the strong confinement in the axial direction the sound speed of the system is $c=\left(1 / 2^{1 / 4}\right) c_{0}$, where $c_{0}$ is the corresponding value without the trap. From the $3 \mathrm{D}$ order-parameter equation of the condensate, we derive a soliton-bearing Kadomtsev-Petriashvili equation with positive dispersion. When the trapping potential is weak in two transverse directions, a low-depth plane dark soliton can propagate in the condensate with a changing profile but preserving its structure down to the boundary of the condensate. We show that high-depth plane dark solitons are unstable to long-wavelength transverse disturbances. The instability appears as a longitudinal modulation of the soliton amplitude decaying into vortices. We also show how a dark lumplike 2D nonlinear excitation can be excited in the system. Furthermore, a dark lump decaying algebraically in two spatial directions can propagate rather stable in the condensate, but disappears near the boundary of the condensate where two vortices are nucleated. The vortices move in opposite directions along the boundary and when meeting merge creating a new lump. Finally, we also provide results for head-on and oblique collisions of two lumps in the system.
\end{abstract}

DOI: 10.1103/PhysRevA.67.023604

PACS number(s): 03.75.Lm, 03.65.Ge, 42.65.Tg

\section{INTRODUCTION}

The recent experimental evidence of Bose-Einstein condensation in weakly interacting atomic gases $[1,2]$ has lead to great progress in atom optics. In particular, it has enabled its extension from the linear to the nonlinear regime, very much like the laser did to nonlinear optics in the 1960s. Macroscopically excited Bose-Einstein condensed states, e.g., solitons and vortices, have been observed [3-8] and four-wave mixing phenomena in a Bose-Einstein condensate (BEC) has also been realized [9]. These studies have, indeed, stimulated research activities in nonlinear atom optics [10].

To our knowledge, most of the works on soliton dynamics in BECs have been limited to one-dimensional (1D) solitons moving in elongated cigar-shaped and quasi-1D periodicshaped traps [11-14], or plane solitons (decaying only in one spatial direction and hence essentially 1D) in a 3D trap $[4,6,14,15]$. For the case of repulsive atom-atom interaction, as normally encountered in BEC experiments, the excitations are dark solitons, that is, "dips" or depression waves in the density profile of the condensate. Such 1D matter-wave dark solitons have been investigated both in theory and in experiment. For 1D dark solitons in the BECs, let us mention the Boussinesq-Korteweg-de Vries description and the study on dark soliton collisions using the Poincaré-Lighthill-Kuo method [16-19].

Although elongated cigar-shaped traps have been widely used in BEC experiments, a flat disk-shaped trap has also been employed [20,21]. In fact, the JILA trap, which was used by Anderson et al. [20] for the first experimental observation of the Bose-Einstein condensation of weakly interacting Bose gases, is just of this type. Later on a disk-shaped trap was also used by Jin et al. [21] to investigate the phononlike linear excitations in the BECs. If the thickness of the disk is small enough, the condensate becomes quasi-2D. One expects that at sufficiently low temperature, the motion of atoms in the direction perpendicular to the disk is frozen and governed by the ground-state wave function in that direction [22]. Such quasi-2D BEC has recently been realized experimentally by Görlitz et al. [23]. As indicated in Ref. [23], the quasi-low-dimensional condensates can provide many opportunities to study the low-dimensional nonlinear excitations in the BECs. Motivated by this important experimental achievement, in the present paper we address the problem of possible 2D soliton excitations in a quasi-2D BEC. We show that, with repulsive atom-atom interaction, weak nonlinear excitations in the condensate evolve according to a Kadomtsev-Petriashvili equation with positive dispersion, which admits lump solutions, i.e., 2D solitons decaying algebraically in all spatial directions. Thus we expect that dark lumplike 2D nonlinear localized excitations are possible in the BECs with disk-shaped traps.

The paper is organized as follows. In Sec. II, we carry on the asymptotic expansion for the Gross-Pitaevskii (GP) equation for the order parameter and derive a KadomtsevPetriashvili (KP) equation for long wavelength, weakly nonlinear excitations. Soliton solutions and the properties of the corresponding excitations in the condensate are discussed. In Sec. III, we cross-check the theoretical predictions by numerical experiments. The transverse stability of a plane (or line) dark soliton and the evolution of a dark lump are thoroughly investigated. Collisions between two lumps are also considered. Finally, Sec. IV contains a discussion and a summary of the results obtained. 


\section{ASYMPTOTIC EXPANSION AND KP-I EQUATION}

\section{A. The model}

The dynamic behavior of a weakly interacting Bose gas at low temperature is described by the time-dependent GP equation [1]

$$
i \hbar \frac{\partial \Psi}{\partial t}=\left[-\frac{\hbar^{2}}{2 m} \nabla^{2}+V_{\text {ext }}(\mathbf{r})+g|\Psi|^{2}\right] \Psi,
$$

where $\Psi$ is the order parameter, $\int d \mathbf{r}|\Psi|^{2}=N$ is the number of atoms in the condensate, $g=4 \pi \hbar^{2} a_{s} / m$ is the interaction constant with $a_{s}$ the $s$-wave scattering length $\left(a_{s}>0\right.$ for repulsive interactions). We consider an anisotropic harmonic trap of the form

$$
V_{\mathrm{ext}}(\mathbf{r})=\frac{m}{2}\left[\omega_{\perp}^{2}\left(x^{2}+y^{2}\right)+\omega_{z}^{2} z^{2}\right],
$$

where $\omega_{\perp}$ and $\omega_{z}$ are frequencies of the trap in the transverse $(x$ and $y)$ directions and in the axial $(z)$ direction, respectively. The choice of an anisotropic trap has been used not only to provide evidence of Bose-Einstein condensation [1], but also for realizing the condensate transition from higher to lower dimensions [23].

Expressing the order parameter in terms of its modulus and phase, $\Psi=\sqrt{n} \exp (i \phi)$, we obtain a set of coupled equations for $n$ and $\phi$. By suitably changing scales and variables, $(x, y, z)=a_{z}\left(x^{\prime}, y^{\prime}, z^{\prime}\right), \quad t=\omega_{z}^{-1} t^{\prime}, \quad n=n_{0} n^{\prime} \quad$ with $a_{z}$ $=\left[\hbar /\left(m \omega_{z}\right)\right]^{1 / 2}$ and $n_{0}=N / a_{z}^{3}$, we arrive at the following dimensionless equations of motion:

$$
\begin{gathered}
\frac{\partial n}{\partial t}+\nabla \cdot(n \boldsymbol{\nabla} \phi)=0 \\
\frac{\partial \phi}{\partial t}+\frac{1}{2} z^{2}+V_{\|}(x, y)+Q n+\frac{1}{2}\left[(\boldsymbol{\nabla} \phi)^{2}-\frac{1}{\sqrt{n}} \nabla^{2} \sqrt{n}\right]=0,
\end{gathered}
$$

with $Q=4 \pi N a_{s} / a_{z}$ (dimensionless interaction constant) and $\int d \mathbf{r} n=1 . V_{\|}(x, y)=\left(\omega_{\perp} / \omega_{z}\right)^{2}\left(x^{2}+y^{2}\right) / 2$ is the dimensionless trapping potential in the $x$ and $y$ directions. Expecting no confusion in the reader we have omitted primes. The last term, i.e., $-\left(\nabla^{2} \sqrt{n}\right) /(2 \sqrt{n})$, in Eq. (4) is the quantum pressure providing the necessary dispersion for forming a soliton in the system, as will be seen below.

We are interested in the excitation created in the condensate with a thin disk-shaped trap. The thin disk-shaped trap here implies that the conditions $a_{z} \ll l_{0}$ and $\hbar \omega_{\perp} \ll n_{0} g$ $\ll \hbar \omega_{z}$ can be fulfilled, where $l_{0}=\left(4 \pi n_{0} a_{s}\right)^{-1 / 2}$ is the healing length. In this situation three consequences follow.

(i) The energy-level spacing in the $z$ direction exceeds largely the interaction energy between atoms, and hence the condensate is quasi-2D. Thus at sufficiently low temperature the motion of atoms in the $z$ direction is essentially frozen and is governed by the ground-state wave function (zeropoint oscillation) of the corresponding harmonic oscillator $[22,23]$. (ii) Due to the strong confinement in the $z$ direction, the excitations can propagate only within the disk plane (i.e., in the $x$ and $y$ directions), similar to a capillary-gravity wave propagating in the surface of a liquid layer [24,25] or an electromagnetic wave propagating along a planar waveguide [26]. Consequently, the superfluid velocity $\mathbf{v}(=\boldsymbol{\nabla} \phi)$ has only $x$ and $y$ components, and hence $\phi=\phi(x, y, t)$.

(iii) As the dimensionless ratio of the trapping frequencies, $\omega_{\perp} / \omega_{z}$, is small, the third term on the left-hand side of Eq. (4) is also a small quantity.

Based on the above considerations, we set

$$
\sqrt{n}=A(x, y, t) G_{0}(z), \quad \phi=-\mu t+\varphi(x, y, t),
$$

or equivalently

$$
\begin{gathered}
\Psi(x, y, z, t)=G_{0}(z) \psi(x, y, t), \\
\psi(x, y, t)=A(x, y, t) \exp [-i \mu t+i \varphi(x, y, t)],
\end{gathered}
$$

where $G_{0}(z)=\exp \left(-z^{2} / 2\right)$ is the ground-state wave function of the 1D harmonic oscillator with the potential $z^{2} / 2$ in the $z$ direction, $\mu$ is the chemical potential of the condensate and $\varphi$ is a phase function contributed from the excitation, which is assumed to be a function of $x$ and $y$ because as mentioned above the excitation can only propagate in the $x$ and $y$ directions.

Thus, Eqs. (3) and (4) can be reduced to

$$
\begin{gathered}
\frac{\partial A}{\partial t}+\frac{\partial A}{\partial x} \frac{\partial \varphi}{\partial x}+\frac{\partial A}{\partial y} \frac{\partial \varphi}{\partial y}+\frac{A}{2}\left(\frac{\partial^{2} \varphi}{\partial x^{2}}+\frac{\partial^{2} \varphi}{\partial y^{2}}\right)=0 \\
-\frac{1}{2}\left(\frac{\partial^{2} A}{\partial x^{2}}+\frac{\partial^{2} A}{\partial y^{2}}\right)-\left(\mu-\frac{1}{2}\right) A+\left[\frac{\partial \varphi}{\partial t}+V_{\|}(x, y)+\frac{1}{2}\left(\frac{\partial \varphi}{\partial x}\right)^{2}\right. \\
\left.+\frac{1}{2}\left(\frac{\partial \varphi}{\partial y}\right)^{2}\right] A+Q^{\prime} A^{3}=0
\end{gathered}
$$

where $Q^{\prime}=I_{0} Q$ is an effective interaction constant with $I_{0}$ $=\int_{-\infty}^{\infty} d z G_{0}^{4}(z) / \int_{-\infty}^{\infty} d z G_{0}^{2}(z)=1 / \sqrt{2}$. To arrive at Eq. (9) we have multiplied Eq. (4) by $G_{0}^{*}$ and then integrated once with respect to $z$ to eliminate the dependence on $z$. A similar approach has been widely used for quasi-1D (cigar-shaped) BEC problems [11,27-32]. In principle, one can take into account the contribution of the higher-order eigenmodes of the harmonic oscillator in the $z$ direction, as done in Ref. [19] for a cigar-shaped trap. However, as here we have assumed $n_{0} g \ll \hbar \omega_{z}$, the contribution from these higher-order eigenmodes is small and can be safely neglected. Furthermore, for the thin disk-shaped trap $\left(\omega_{\perp} / \omega_{z} \ll 1\right)$ the trapping potential in the $(x, y)$ plane is a slowly varying function of $x$ and $y$ and hence the size of the condensate in the radial direction is much larger than the size of the soliton excitations (with the order of the healing length) considered below. In the propagation of the soliton at short times, the boundary of the condensate does not come into play and we can therefore simulate the experimental situation by considering the condensate being uniform in the $(x, y)$ plane. On the other hand, in order 
to get insight on the possible 2D soliton excitations as a first step we disregard the slowly varying radial trapping potential for the analytical approach given below. However, when doing numerics in Sec. III we shall include this term, thus cross-checking the validity of the approximation used in the present section. A treatment equivalent to the local-density approximation when including the slowly varying trapping potential in the $(x, y)$ plane is described in the last part of Sec. II C.

\section{B. Asymptotic expansion and KP-I equation}

We now begin to study the possible weak nonlinear excitations in a disk-shaped condensate. Before doing this it is useful to discuss linear excitations and, in particular, the linear dispersion relation (excitation spectrum) of the system. Taking $\quad A=u_{0}+a(x, y, t) \quad\left(u_{0}>0\right) \quad$ with $\quad(a, \varphi)$ $=\left(a_{0}, \varphi_{0}\right) \exp \left[i\left(k_{1} x+k_{2} y-\omega t\right)\right]+$ c.c. (c.c. denotes complex conjugate), assuming that $a_{0}$ and $\varphi_{0}$ are small constants, and linearizing Eqs. (8) and (9), one obtains $\mu=1 / 2+Q^{\prime} u_{0}^{2}$ and

$$
\omega\left(k_{1}, k_{2}\right)=\frac{1}{2} k\left(4 Q^{\prime} u_{0}^{2}+k^{2}\right)^{1 / 2},
$$

where $k=\left(k_{1}^{2}+k_{2}^{2}\right)^{1 / 2}$ is the wave number and $\omega$ is the frequency of the excitation. Equation (10) corresponds to a Bogoliubov-type linear excitation spectrum in twodimensions. We see that to obtain the Bogoliubov excitation spectrum, the atom-atom interaction (represented by $Q^{\prime}$ ) and the quantum pressure [reflected by the $k^{2}$ term in the bracket of Eq. (10)] are needed. Another notable feature of the excitation spectrum is that the system allows a long-wavelength (i.e., sound) excitation. The sound speed of the system is given by

$$
c=\lim _{k \rightarrow 0}\left[\left(\frac{\partial \omega}{\partial k_{1}}\right)^{2}+\left(\frac{\partial \omega}{\partial k_{2}}\right)^{2}\right]^{1 / 2}=\sqrt{Q^{\prime}} u_{0} .
$$

Note that for a homogeneous system [i.e., $V_{\text {ext }}(\mathbf{r})=0$ ] the corresponding sound speed in our notation is $c_{0}=\sqrt{Q} u_{0}$. Thus we have

$$
\frac{c}{c_{0}}=\sqrt{\frac{Q^{\prime}}{Q}}=\frac{1}{2^{1 / 4}}
$$

The decrease of the sound speed relative to the homogeneous system is due to the confinement of the system in the $z$ direction. This value of the sound speed in the disk-shaped BEC is higher than the corresponding value in a cigar-shaped trap, where the confinement occurs in two directions [18].

Let us now investigate the onset of weakly nonlinear excitations in the system. For a long-wavelength excitation we set $A=u_{0}+\epsilon^{2}\left(a^{(0)}+\epsilon^{2} a^{(1)}+\cdots\right)$ and $\varphi=\epsilon\left(\varphi^{(0)}+\epsilon^{2} \varphi^{(1)}\right.$ $+\cdots)$, where $a^{(j)}$ and $\varphi^{(j)}(j=0,1, \ldots)$ are functions of the multiple-scale (slow) variables $\xi=\epsilon\left(c^{-1} x-t\right), \quad \eta$ $=\epsilon^{2} y$, and $\tau=\epsilon^{3} t$, with $\epsilon$ a smallness and ordering parameter characterizing the relative amplitude of the excitation. Substituting the expansion into Eqs. (8) and (9) we obtain

$$
\begin{aligned}
& \frac{\partial a^{(j)}}{\partial \xi}-\frac{1}{2 c^{2}} u_{0} \frac{\partial^{2} \varphi^{(j)}}{\partial \xi^{2}}=\alpha^{(j)}, \\
& 2 Q^{\prime} u_{0}^{2} a^{(j)}-u_{0} \frac{\partial \varphi^{(j)}}{\partial \xi}=\beta^{(j)},
\end{aligned}
$$

for $j=0,1, \ldots$ The explicit expressions of $\alpha^{(j)}$ and $\beta^{(j)}$ are omitted here [33].

In the leading order $(j=0)$, we obtain

$$
\varphi^{(0)}=\left(2 c^{2} / u_{0}\right) \int d \xi a^{(0)},
$$

with $a^{(0)}$ a function yet to be determined. The solvability condition in this order requires $c=\sqrt{Q^{\prime}} u_{0}$, which is just the sound speed of the system. At the next order $(j=1)$, the solvability condition results in a closed equation for $a^{(0)}$ :

$\frac{\partial}{\partial \xi}\left(\frac{\partial a^{(0)}}{\partial \tau}+\frac{3 \sqrt{Q^{\prime}}}{c} a^{(0)} \frac{\partial a^{(0)}}{\partial \xi}-\frac{1}{8 c^{2}} \frac{\partial^{3} a^{(0)}}{\partial \xi^{3}}\right)+\frac{c^{2}}{2} \frac{\partial^{2} a^{(0)}}{\partial \eta^{2}}=0$.

Equation (16) is the soliton-bearing KP equation [25]. We see that the dispersion term (i.e., the fourth-order derivative term with respect to $\xi$ ) comes from the quantum pressure of the system. Note that Eq. (16) is a positive-dispersion KP equation (also called the KP-I equation) since the dispersion term and the diffractive term (i.e., the second-order derivative term with respect to $\eta$ ) have opposite signs $[34,35]$. The KP-I equation is a completely integrable system and can be solved by the inverse scattering transform [35].

\section{2D soliton solutions}

In this section, we give 2D soliton solutions of the KP-I equation derived above in the form of dark 2D soliton excitations in the disk-shaped BEC. Changing variables once more, $\quad \epsilon^{2} a^{(0)}=-\left(u_{0} / 4 c^{2}\right) U, \quad X^{\prime}=-X=-(x-c t), \quad y^{\prime}$ $=(\sqrt{3} / 2 c) y$, and $t^{\prime}=(1 / 8 c) t$, Eq. (16) reads

$$
\frac{\partial}{\partial X^{\prime}}\left(\frac{\partial U}{\partial t^{\prime}}+6 U \frac{\partial U}{\partial X^{\prime}}+\frac{\partial^{3} U}{\partial X^{\prime} 3}\right)-3 \frac{\partial^{2} U}{\partial y^{\prime 2}}=0
$$

which is one of the standard forms of the KP-I equation.

The KP-I Eq. (17) allows different types of soliton solutions. One of them is a plane soliton given by

$$
U=2 p^{2} \operatorname{sech}^{2}\left\{p\left[X^{\prime}+q y^{\prime}-\left(4 p^{2}-3 q^{2}\right) t^{\prime}-X_{0}\right]\right\},
$$

where $p, q$, and $X_{0}$ are arbitrary constants. Note that the plane-soliton solution (18) is localized only in its traveling direction. From Eq. (15) one obtains

$$
\epsilon \varphi^{(0)}=-\frac{p}{c} \tanh \left\{p\left[X^{\prime}+q y^{\prime}-\left(4 p^{2}-3 q^{2}\right) t^{\prime}-X_{0}\right]\right\} .
$$


Thus, up to the first-order approximation, the order parameter of the condensate corresponding to the plane-soliton solution is

$$
\begin{aligned}
\Psi= & u_{0}\left(1-\frac{p^{2}}{2 c^{2}} \operatorname{sech}^{2}\left\{p \left[x-\frac{\sqrt{3} q}{2 c} y\right.\right.\right. \\
& \left.\left.\left.-c\left(1-\frac{p^{2}}{2 c^{2}}+\frac{3 q^{2}}{8 c^{2}}\right) t-x_{0}\right]\right\}\right) \exp \left(-z^{2}-i \mu t-i \frac{p}{c}\right. \\
& \left.\times \tanh \left\{p\left(x-\frac{\sqrt{3} q}{2 c} y-c\left(1-\frac{p^{2}}{2 c^{2}}+\frac{3 q^{2}}{8 c^{2}}\right) t-x_{0}\right]\right\}\right) .
\end{aligned}
$$

This is a dark plane soliton traveling in the direction defined by the vector $(\mathbf{i},-\sqrt{3} q /(2 c) \mathbf{j})$ with the velocity

$$
v_{\mathrm{ps}}=c \frac{1-\frac{p^{2}}{2 c^{2}}+\frac{3 q^{2}}{8 c^{2}}}{\sqrt{1+\frac{3 q^{2}}{4 c^{2}}}},
$$

which is lower than the sound velocity, $c$, in the system. The parameter $p$ reflects the grayness of the soliton $\left(0 \leqslant p^{2}\right.$ $\left.\leqslant 2 c^{2}\right)$. As we shall see in the following section, highly deep enough dark plane solitons are unstable to long-wavelength transverse disturbances.

The KP-I Eq. (17) also admits 2D lumps decaying algebraically in all directions [35]. A single-lump solution of Eq. (17) is given by

$$
U=\frac{4 \nu\left[1-\nu\left(X^{\prime}-3 \nu t^{\prime}\right)^{2}+\nu^{2}\left(y^{\prime}\right)^{2}\right]}{\left[1+\nu\left(X^{\prime}-3 \nu t^{\prime}\right)^{2}+\nu^{2}\left(y^{\prime}\right)^{2}\right]^{2}},
$$

where $\nu$ is an arbitrary positive constant. In the leading-order approximation, the phase of the order parameter is

$$
\epsilon \varphi^{(0)}=-\frac{2 \nu}{c} \frac{X^{\prime}-3 \nu t^{\prime}}{1+\nu\left(X^{\prime}-3 \nu t^{\prime}\right)^{2}+\nu^{2}\left(y^{\prime}\right)^{2}} .
$$

Returning to the original variables we obtain the order parameter of the condensate when the lump is created:

$$
\begin{aligned}
& \Psi=u_{0}\left\{1-\frac{\nu}{c^{2}} \frac{1-\nu\left(x^{\prime}\right)^{2}+\frac{3 \nu^{2}}{4 c^{2}} y^{2}}{\left[1+\nu\left(x^{\prime}\right)^{2}+\frac{3 \nu^{2}}{4 c^{2}} y^{2}\right]^{2}}\right\} \\
& \times \exp \left\{-z^{2}-i \nu t+i \frac{2 \nu}{c} \frac{x^{\prime}}{1+\nu\left(x^{\prime}\right)^{2}+\frac{3 \nu^{2}}{4 c^{2}} y^{2}}\right\} \text {, }
\end{aligned}
$$

where $x^{\prime}=x-c\left[1-3 \nu /\left(8 c^{2}\right)\right] t$ and hence the velocity of the lump is given by

$$
v_{\text {lump }}=c\left(1-\frac{3 \nu}{8 c^{2}}\right)
$$

Thus the lump, characterized by the background parameter $u_{0}$ and the grayness parameter $\nu$, is also a subsonic excitation. Hence all solitons here are subsonic. This originates in the repulsive character of the atom-atom interaction.

From Eq. (17) one can also get a 1D (periodic) lump solution [36]

$$
U=2 p^{2} \frac{1+\sqrt{1-\frac{p^{4}}{k_{y}^{2}}} \cosh \left(p X^{\prime}-\omega t^{\prime}+\delta\right) \cos \left(k_{y} y\right)}{\left[\cosh \left(p X^{\prime}-\omega t^{\prime}+\delta\right)+\sqrt{1-\frac{p^{4}}{k_{y}^{2}}} \cos \left(k_{y} y\right)\right]^{2}},
$$

where $\omega=p^{3}+3 k_{y}^{2} / p$ with $p, k_{y}$, and $\delta$ being integration constants. The order parameter in this case is given by

$$
\begin{aligned}
\Psi= & u_{0}\left[1-\frac{p^{2}}{2 c^{2}} \frac{1+\sqrt{1-\frac{p^{4}}{k_{y}^{2}}} \cosh x^{\prime} \cos \left(\frac{\sqrt{3} k_{y}}{2 c} y\right)}{\left.\cosh x^{\prime}+\sqrt{1-\frac{p^{4}}{k_{y}^{2}}} \cos \left(\frac{\sqrt{3} k_{y}}{2 c} y\right)\right]^{2}}\right] \\
& \times \exp \left[-z^{2}-i \mu t+\frac{i p}{c}\right. \\
& \left.\times \frac{\sinh x^{\prime}}{\sqrt{1-\frac{p^{4}}{k_{y}^{2}}} \cos \left(\frac{\sqrt{3} k_{y}}{2 c} y\right)}\right],
\end{aligned}
$$

where $x^{\prime}=p x-c\left(p-\omega / 8 c^{2}\right) t-\delta$. The 1D dark lump represented by the solution (27) has the same subsonic velocity $c\left(1-\omega / 8 c^{2} p\right)$ along the $x$ direction.

From the results presented above we can say that the BEC with disk-shaped trap and repulsive atom-atom interaction may be a realistic physical system for observing highdimensional nonlinear localized excitations. The formation of the 2D solitons is due to the balance between the dispersion provided by kinetic energy and the nonlinearity coming from the atom-atom interaction.

To justify the approximation (5) we note that in the $z$ direction the trapping potential tends to compress the order parameter competing against the nonlinear force as well as the linear dispersion effect provided by the kinetic energy. On the other hand, the trapping force in the $x$ and $y$ directions is small (since $\omega_{\perp} / \omega_{z} \ll 1$ ), so that along the $x$ and $y$ direc- 
tions effects come only from the nonlinear force and the dispersion. If the main force in the $z$ direction is caused by the trapping potential, the approximation given by Eqs. (5) follows. Notice that the dimensionless energy functional of Eq. (1) is given by

$$
E=\int \operatorname{dxdydz}\left(\mathcal{H}_{\text {kin }}+\mathcal{H}_{\text {trap }}+\mathcal{H}_{\text {int }}\right),
$$

with

$$
\begin{gathered}
\mathcal{H}_{\text {kin }}=\frac{1}{2}|\nabla \sqrt{n}|^{2}, \quad \mathcal{H}_{\text {trap }}=\left[\frac{1}{2} z^{2}+V_{\|}(x, y)\right] n, \\
\mathcal{H}_{\text {int }}=\frac{1}{2} Q n^{2},
\end{gathered}
$$

where $\mathcal{H}_{\text {kin }}, \mathcal{H}_{\text {trap }}$, and $\mathcal{H}_{\text {int }}$ denote densities of kinetic energy, trapping potential energy, and self-interaction energy, respectively. All energies have been scaled with the unit $N \hbar \omega_{z}$. To check the approximation (5) let us consider the ratio between the trapping potential in the $z$ direction (i.e., $\left.\mathcal{H}_{z \text {-trap }}=z^{2} n / 2\right)$ and the self-interaction energy, $\mathcal{H}_{\text {int }}$, for the above given solution. All solutions for $A(x, y, t)$ have the form $A=u_{0}+\epsilon^{2} a^{(0)}+O\left(\epsilon^{4}\right)$. Thus we get

$$
R=\frac{z^{2} \exp \left(z^{2}\right)}{Q u_{0}^{2}\left[1+\epsilon^{2} a^{(0)}+\cdots\right]^{2}} \approx \frac{z^{2} \exp \left(z^{2}\right)}{Q u_{0}^{2}} .
$$

As $Q u_{0}^{2}$ is a constant we have $R \gg 1$, except for small values of $z$. Thus the parabolic trapping potential in the $z$ direction dominates over the self-interaction energy and then the only effect of the nonlinearity on the shape of the order parameter in the $z$ direction is to provide a small correction near the center of the trap, which is the place where the parabolic potential is the lowest and the nonlinear effect is more relevant. Consequently, when the condition $n_{0} g \ll \hbar \omega_{z}$ is satisfied, the approximation (5) is justified.

If the effect of the slowly varying trapping potential in the $(x, y)$ plane is taken into account, Eq. (16) should be replaced by a modified Kadomtsev-Petriashvili (MKP) equation

$$
\begin{gathered}
\frac{\partial}{\partial \xi}\left(\frac{\partial a^{(0)}}{\partial \tau}+\frac{3 \sqrt{Q^{\prime}}}{c} a^{(0)} \frac{\partial a^{(0)}}{\partial \xi}-\frac{1}{8 c^{2}} \frac{\partial^{3} a^{(0)}}{\partial \xi^{3}}\right)+\frac{c^{2}}{2} \frac{\partial^{2} a^{(0)}}{\partial \eta^{2}} \\
\quad=-\delta_{i 3} \frac{\partial}{\partial \xi}\left(\frac{3}{2} \frac{\partial c}{\partial x_{i}} a^{(0)}+c \frac{\partial a^{(0)}}{\partial x_{i}}\right)
\end{gathered}
$$

where

$$
c=\sqrt{Q^{\prime}} u_{0}\left(x_{i}, y_{i}\right)
$$

is local sound speed, and

$$
u_{0}=\left\{\left[\mu-1 / 2-V_{\|}\left(x_{i}, y_{i}\right)\right] / Q^{\prime}\right\}^{1 / 2}
$$

is the ground-state configuration of the condensate in the $x$ and $y$ directions, with $x_{i}=\epsilon^{i} x$ and $y_{i}=\epsilon^{i} y \quad(i \geqslant 3)$. Here $V_{\|}(x, y)$ is basically an arbitrary (slowly varying) function.
For a harmonic potential one has $V_{\|}=\left(\omega_{\perp} / \omega_{z}\right)^{2}\left(x^{2}+y^{2}\right) / 2$ $=\Omega_{0}^{2}\left(x_{i}^{2}+y_{i}^{2}\right) / 2$ under the assumption $\omega_{\perp} / \omega_{z}=\epsilon^{i} \Omega_{0}$, with $\Omega_{0}$ a constant of order unity. A detailed derivation of Eq. (31) is given in the Appendix.

If the trapping potential in the $(x, y)$ plane varies very slowly, i.e., if $V_{\|}(x, y)=V_{\|}\left(x_{i}, y_{i}\right)$ with $i \geqslant 4$, the terms on the right-hand side of Eq. (31) disappear and hence Eq. (31) takes the same form of the KP Eq. (16), but the quantity $c$ is now a local sound speed (32). In this case the soliton solutions (20), (24), and (27) are still valid, but $c$ should be replaced by the local sound speed. Such an approach is equivalent to the local-density approximation. Even in the case of $i=3$ the MKP Eq. (31) can be approximated as a KP equation in the region far from the boundary of the condensate. Indeed, far from the boundary the effect of the trapping potential on the evolution of the soliton is negligible.

Because of the variable coefficients and the additional terms, an analytical approach of the MKP Eq. (31) is not an easy task. One expects that the 2D solitons (20), (24), and (27) will deform and even be unstable, and hence we turn to a numerical simulation in the following section.

\section{NUMERICAL SIMULATIONS}

In this section, we give numerical evidence for the existence of the various solutions presented in the preceding section and cross-check their stability according to Eqs. (8) and (9). As earlier stated, in the computer experiments we shall take into account the trapping potential in both $x$ and $y$ directions. We assume that in Eq. (9),

$$
V_{\|}(x, y)=\frac{1}{2}\left(\frac{\omega_{\perp}}{\omega_{z}}\right)^{2}\left(x^{2}+y^{2}\right) A .
$$

For convenience we again change variables,

$$
\begin{gathered}
A=2^{-1 / 4} \sqrt{\frac{\omega_{\perp}}{\omega_{z} Q}} \rho, \quad t=\frac{2 \omega_{z}}{\omega_{\perp}} \tau, \\
x=\sqrt{\frac{\omega_{z}}{\omega_{\perp}}} s_{1}, \quad y=\sqrt{\frac{\omega_{z}}{\omega_{\perp}}} s_{2},
\end{gathered}
$$

where $s_{1}$ and $s_{2}$ denote new Cartesian coordinates, $\tau$ is the new time, and $\rho$ is a new quantity proportional to the amplitude of the order parameter. Then Eqs. (8) and (9) become

$$
\frac{\partial \rho}{\partial \tau}=-2(\nabla \rho)(\nabla \varphi)-\rho \nabla^{2} \varphi
$$

$$
\rho \frac{\partial \varphi}{\partial \tau}=\nabla^{2} \rho-\left[s_{1}^{2}+s_{2}^{2}-l^{2}+\rho^{2}+(\boldsymbol{\nabla} \varphi)^{2}\right] \rho,
$$

where the spatial derivatives are taken with respect to $\left(s_{1}, s_{2}\right) ; l^{2}=\omega_{z}(2 \mu-1) / \omega_{\perp}$. The constant $l$ in Eqs. (35) and (36) defines the length scale in the problem (i.e., the dimensionless radius of the condensate). We assume that the particle number in the condensate is large enough and hence 


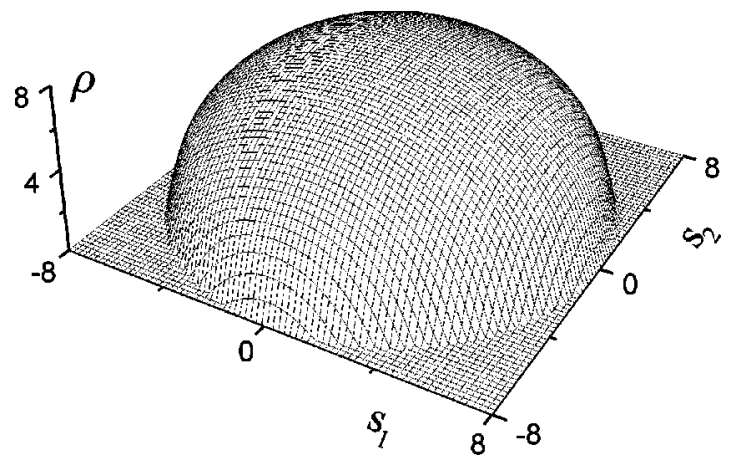

FIG. 1. Stationary background profile of the condensate ( $l$ $=8$ ). Note that all quantities appearing in this and the following figures are dimensionless.

on the boundary, where $s_{1}^{2}+s_{2}^{2}=l^{2}$, the order parameter approximately vanishes. Accordingly, at the boundary we have $\rho=\varphi^{\prime}=0$.

Here, since $\omega_{\perp} / \omega_{z} \ll 1$, the chemical potential $\mu$ of the system can be estimated using the Thomas-Fermi approximation for the ground-state wave function in the $x$ and $y$ directions. We get

$$
\mu=\frac{1}{2}+\frac{\omega_{\perp}}{\omega_{z}}\left(\frac{I_{0} Q}{\pi^{3 / 2}}\right)^{1 / 2},
$$

measured in $\hbar \omega_{z}$ units. The relationship (37) supports the quasi-2D criterion that the additional energy due to the atomatom interaction is much less than the characteristic energy scale in the $z$ direction, i.e., $\mu-\hbar \omega_{z} / 2 \ll \hbar \omega_{z} / 2$ (with physical units) and hence justifies again the assumption underlying Eqs. (5).

Now let us find the shape of the stationary background state, which has been taken to be approximately flat for the analytical study in the preceding section. Since in the ground state, the phase $\varphi_{0}=$ constant, we get from Eqs. (35) and (36) the time-independent, nonlinear equation for $\rho_{0}$,

$$
\frac{d^{2} \rho_{0}}{d r^{2}}=\left(r^{2}-l^{2}+\rho_{0}^{2}\right) \rho_{0},
$$

where $r=\sqrt{s_{1}^{2}+s_{2}^{2}}$ denotes the radius in polar coordinates. Figure 1 shows the background pedestal profile, i.e., solution of Eq. (38) with $l=8$ used in all subsequent computer calculations.

\section{A. Propagation of dark plane solitons}

Let us now search for the dark plane-soliton solution when including the slowly varying trapping potential in the $(x, y)$ plane. We use the soliton solution obtained in the preceding section as an initial condition. From Eq. (20) by putting $t=0$ we get the initial condition that will be used to integrate Eqs. (35) and (36):

$$
\begin{gathered}
\rho=\rho_{0}\left(1-\varepsilon_{1} \operatorname{sech}^{2} \theta\right), \quad \varphi=-\sqrt{2 \varepsilon_{1}} \tanh \theta, \\
\theta=\sqrt{\varepsilon_{1}} \rho_{0}^{*}\left(s_{1}-s_{1}^{0}-\varepsilon_{2} s_{2}\right) .
\end{gathered}
$$
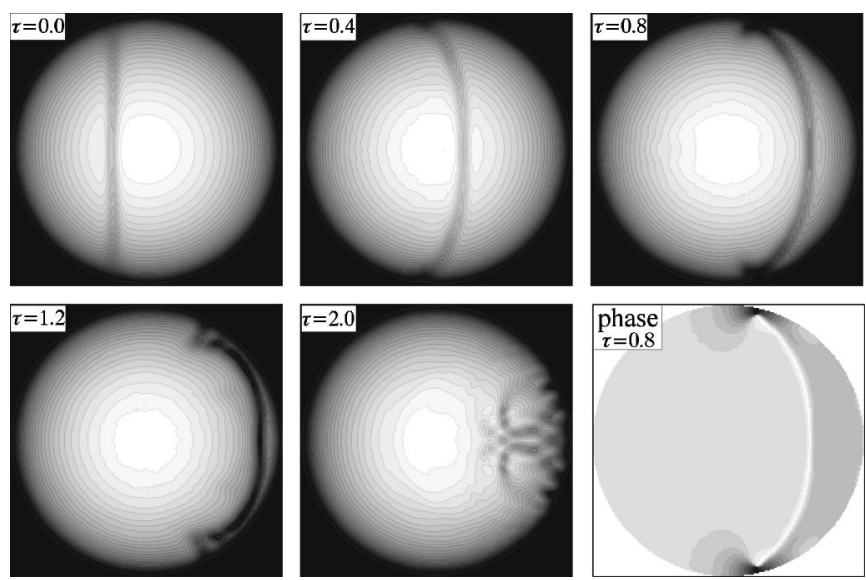

FIG. 2. Sequential snapshots of condensate with low-depth dark plane soliton (brightness corresponds to the amplitude value $\rho\left(s_{1}, s_{2}\right)$; the last snapshot shows the phase $\varphi\left(s_{1}, s_{2}\right)$ where the interval $[-\pi, \pi]$ corresponds to brightness going from black to black via white). The soliton propagates from the left to the right and finally is destroyed due to interaction with the boundary $\left(\varepsilon_{1}\right.$ $=0.2, \varepsilon_{3}=0.05$, and $\left.k_{2}=2 \pi / l\right)$. Note that the relation between the dimensionless time, $\tau$, and the dimensional time, $t$, is $\tau=\omega_{\perp} t / 2$ (which is the same for subsequent figures). Thus for $\omega_{\perp} /(2 \pi)$ $=10 \mathrm{~Hz}$ the time sequence shown in the figure for the amplitude evolution is $0.0 \mathrm{~ms}, 12.8 \mathrm{~ms}, 25.6 \mathrm{~ms}, 38.4 \mathrm{~ms}$, and $64 \mathrm{~ms}$, respectively.

Here $\rho_{0}\left(s_{1}, s_{2}\right)$ is the stationary pedestal (i.e., the condensate density in the ground state of the system). However, to satisfy the boundary conditions, we now use its real profile obtained above (Fig. 1). Hence, we have a dark planelike soliton whose depth changes along the longitudinal direction. Besides, to have a plane phase far away from the soliton location we use $\rho_{0}^{*}=\rho_{0}\left(s_{1}^{0}, s_{2}\right)$. The constant $\varepsilon_{1}=p^{2} / 2 c^{2}$ defines the grayness or soliton depth $\left(\varepsilon_{1}=1\right.$ corresponds to the black soliton). $\varepsilon_{2}$ corresponds to the slope in the longitudinal direction of the soliton in the coordinates $\left(s_{1}, s_{2}\right)$. Due to symmetry we can set $\varepsilon_{2}=0$ (it corresponds to a vertically oriented soliton). To check the stability to transverse perturbations of the dark plane soliton instead of the "perfect" phase we use

$$
\theta=\sqrt{\varepsilon_{1}} \rho_{0}^{*}\left[s_{1}-s_{1}^{0}+\varepsilon_{3} \cos \left(k_{2} s_{2}\right)\right],
$$

where $\varepsilon_{3}$ is a smallness parameter accounting for the strength of the perturbation. Experiments $[6,14,15]$ have shown that dark plane solitons in 3D BECs are unstable to long-wave transverse perturbations. Accordingly, $k_{2}$ was chosen small. In our numerical experiment we used the length, $l$, as a scale factor for $k_{2}$.

Figure 2 shows the results of the numerical integration of Eqs. (35) and (36) with a dark plane soliton of low enough initial depth (around 20\%). Equations (39) and (40) have been used as initial conditions for the integration. In the first five snapshots we show the amplitude $\rho\left(s_{1}, s_{2}\right)$ which is proportional to the density of the condensate, a quantity that can be directly observed in the experiment. The last snapshot shows the phase distribution at $\tau=0.8$. Soon after start, a 
very low (almost negligible) amplitude radiation wave propagating to the left is emitted. The dark plane soliton propagates in the opposite direction with velocity $v_{p s}$ $\approx 8.95$ (measured when it passes over the center of the condensate). The theoretical estimate from Eq. (21) gives

$$
v_{\text {th }}=\sqrt{2} \rho_{0}\left(1-\varepsilon_{1}\right) \approx 9.05,
$$

which is significantly lower than the sound speed ( $c$ $\approx 11.3$ ) and close to the value obtained in the numerical test. By definition (34), in physical units velocity of the plane soliton is $v_{\mathrm{ps}}=\left(a_{z} / 2\right) \sqrt{\omega_{z} \omega_{\perp}} v_{\text {th }}$. Using data from Ref. [23], i.e., $\omega_{z} /(2 \pi)=790 \mathrm{~Hz}$ and $\omega_{\perp} /(2 \pi)=10 \mathrm{~Hz}$ (thus $a_{z}$ $\left.=\left[\hbar /\left(m \omega_{z}\right)\right]^{1 / 2}=0.74 \mu \mathrm{m}\right)$, one obtains that $v_{\mathrm{ps}}$ $=1.87 \mathrm{~mm} \mathrm{~s}^{-1}$ when passing over the center of the condensate, which is less than the sound speed $c=2.33 \mathrm{~mm} \mathrm{~s}^{-1}$. The soliton profile changes during propagation. The curvature of the front can be explained by the dependence of the velocity [Eq. (41)] on the condensate density. The velocity is maximum at the center and decreases down to zero at the boundary, hence central soliton parts move faster and, consequently, a curved front is formed. No transverse instability, at least during the propagation time, occurs here (first four snapshots in Fig. 2). Small perturbations do not grow in time. However, the interaction with the boundary is destructive leading to the appearance of complex wavy excitations $(\tau$ $=2.0$ in Fig. 2).

The first stage of the soliton propagation (until interacting with the boundary) is similar to that observed experimentally [4] in the BEC with a 3D trap by imprinting a phase step (around $1.5 \pi$ ) at the center of the condensate. A dark plane soliton moving from the center to the boundary with a subsonic velocity has been observed in the experiment. The shape of the front changes as illustrated in Fig. 2.

Figure 3 shows the evolution of an initially excited highdepth dark plane-soliton. Except the soliton depth $\left[\epsilon_{1}=0.8\right.$ in Eq. (39)], all parameter values are identical to those used in the calculations leading to Fig. 2. However, the evolution of the soliton is clearly different from that shown in Fig. 2. In this case, we observed a snake instability leading to the nucleation of several vortices, while in the previous case the plane dark soliton has been shown to be stable to transverse perturbations at least for the time up to the contact with the boundary. The nucleation of vortices due to instability of a narrow density defect in a two-component BEC has been reported in an experiment with a 3D trap [6].

Vortices, as they appear in many physical systems, are characterized by phase singularities [37-39], which in our case can be clearly seen in Fig. 3 (phase snapshot). Generally, a vortex is a more stable evolving structure than a plane soliton. The latter tends to collapse like we have observed in the second computer experiment. The instability can be explained as follows. Initial transverse disturbances lead to modulation of the soliton amplitude along the longitudinal direction. Then due to the amplitude dependence of the propagation velocity, those parts of the soliton with relatively lower depth overcome the "normal" propagation process while parts with higher depth slow down (see Fig. 3, $\tau$ $=0.8$ ), hence amplifying the transverse perturbations and
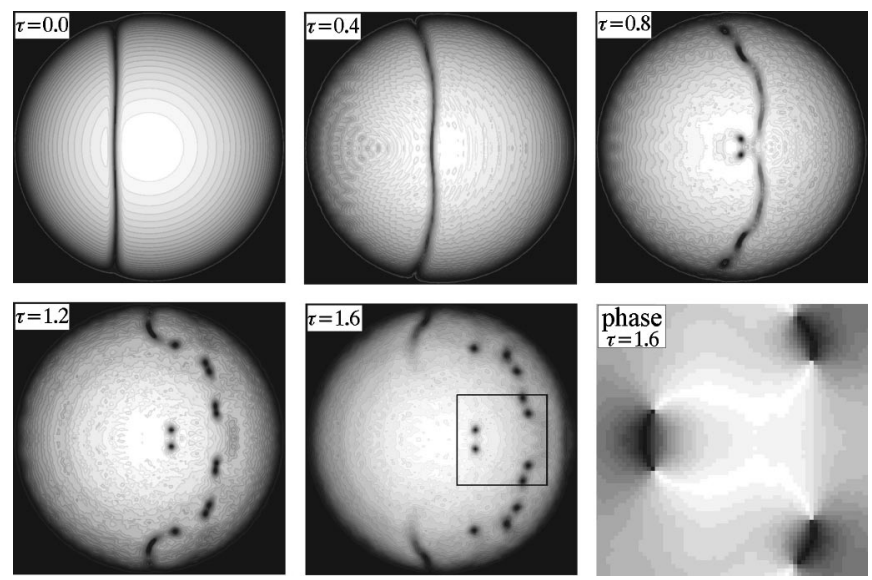

FIG. 3. Sequential snapshots of the condensate with a highdepth dark plane soliton initially excited (initial conditions are similar to those used for Fig. 2). The brightness in the first five snapshots corresponds to the amplitude value $\rho\left(s_{1}, s_{2}\right)$. The soliton propagates from the left to the right with a developing snake instability (snapshot at $\tau=0.4$ ) eventually decaying into vortices (snapshots at $\tau=0.8,1.2$, and 1.6). The last snapshot shows the phase $\varphi\left(s_{1}, s_{2}\right)$ in the square region marked on the corresponding amplitude snapshot for $\tau=1.6$ (the interval $[-\pi, \pi]$ corresponds to brightness going from black to black via white). It includes six vortices with phase singularities. The phase changes by $2 \pi$ along a closed path around each vortex $\left(\varepsilon_{1}=0.8, \varepsilon_{3}=0.05\right.$, and $k_{2}$ $=2 \pi / l)$.

increasing the local curvature. In turn this leads to a higher amplitude modulation and finally the plane soliton tears into pieces. At the initial stage of the instability we have observed the formation of nine pieces, hence a wavelength around $\lambda$ $=8 / 9$. However, in the first run (Fig. 2) the plane dark soliton (it becomes curved due to the inhomogeneity of the system and strictly speaking it is not exactly a plane soliton) propagates up to the boundary keeping its shape. Such difference in the behavior of low-depth solitons may be explained by their relatively higher velocity and limited propagation length (due to boundaries of the condensate), and hence, by insufficient observation time.

\section{B. Propagation and collision of lumps}

Let us now consider the propagation of dark lumps in the system when including the inhomogeneity in $x$ and $y$ directions. From Eq. (24) we get the initial condition for a lump solution,

$$
\begin{gathered}
\rho=\rho_{0}\left\{1-\varepsilon \frac{1+\frac{\varepsilon}{2} \rho_{0}^{2}\left[\frac{3}{4} \varepsilon s_{2}^{2}-\left(s_{1}-s_{1}^{0}\right)^{2}\right]}{\left.\left\{1+\frac{\varepsilon}{2} \rho_{0}^{2}\left[\frac{3}{4} \varepsilon s_{2}^{2}+\left(s_{1}-s_{1}^{0}\right)^{2}\right]\right\}^{2}\right\},}\right. \\
\varphi=\frac{\sqrt{2} \varepsilon \rho_{0}\left(s_{1}-s_{1}^{0}\right)}{1+\frac{\varepsilon}{2} \rho_{0}^{2}\left(\frac{3}{4} \varepsilon s_{2}^{2}+\left(s_{1}-s_{1}^{0}\right)^{2}\right)} .
\end{gathered}
$$



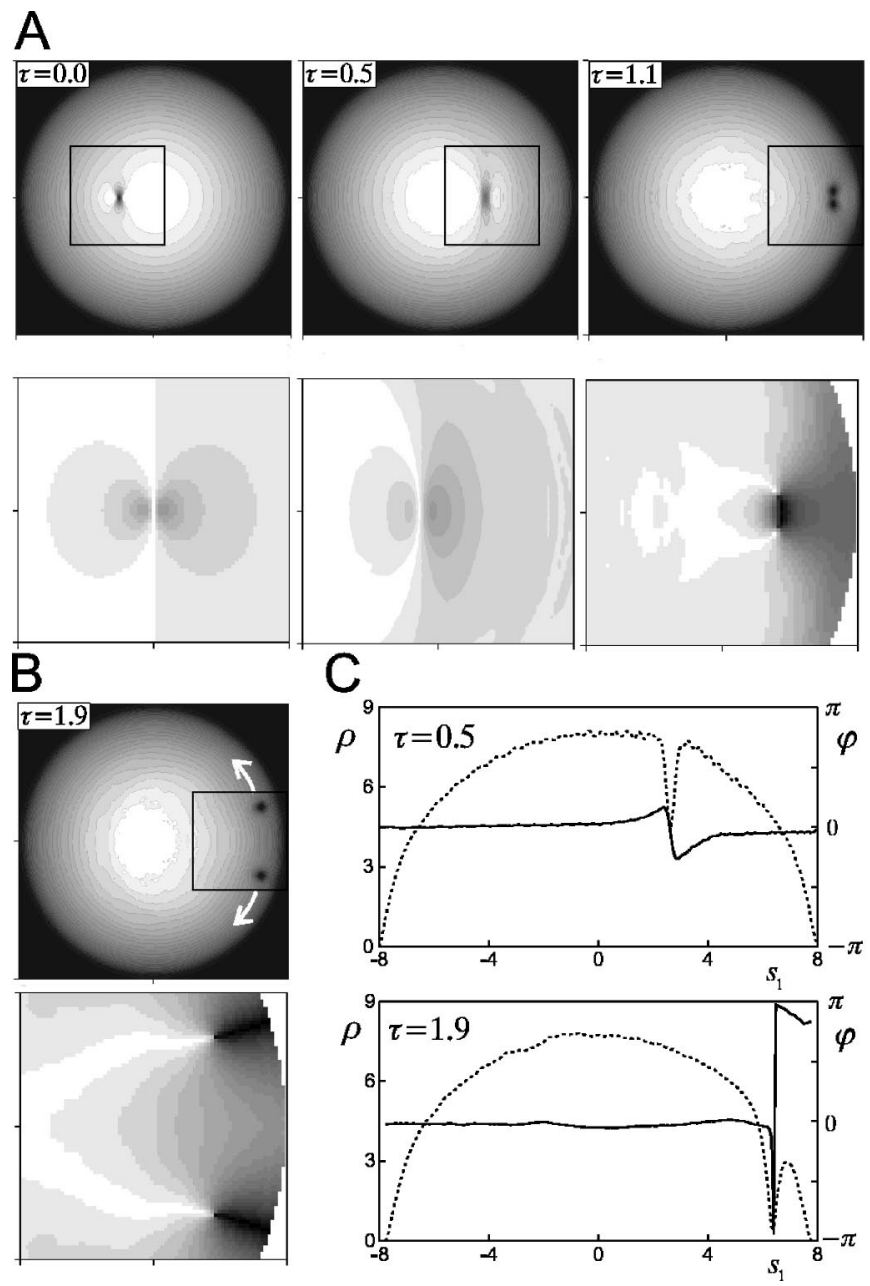

FIG. 4. Propagation of a lump solution and the nucleation near the edge of two moving, clockwise and counterclockwise, vortices $[\varepsilon=0.8$ in Eq. (42)]. (a) Sequential snapshots of the condensate with a high-depth lump initially excited (in the first row brightness corresponds to amplitude value $\rho\left(s_{1}, s_{2}\right)$, in the second row brightness going from black to black via white reflects the phase $\varphi\left(s_{1}, s_{2}\right)$ in the interval $[-\pi, \pi]$ (enlarged square regions are shown)). The lump propagates from the left to the right. Then, near the boundary, its depth approaches $100 \%$, the lump becomes practically black and it breaks into two vortices. (b) The vortices move along the boundary of the condensate in opposite directions (arrows show motion directions). (c) Section of the condensate along the "horizontal" axis $s_{1}$ crossing the lump for $\tau=0.5$ and the vortex for $\tau=1.9$. Solid and dashed lines correspond to the phase and the amplitude, respectively.

Figure 4 shows the time evolution of an initially excited high-depth lump. It represents a localized (in both directions) excitation elongated in $s_{2}$. The initial depth of the lump [ $\varepsilon$ $=0.8$ in Eqs. (42)] is the same as the depth of the soliton shown in Fig. 3. However, during the first propagation stage $(0<\tau \leqslant 0.15)$, the lump emits sound waves and reduces its depth to $\varepsilon_{\text {effec }} \approx 0.45$ thus becoming brighter and wider (see the snapshots for $\tau=0.0$ and 0.5 in Fig. 4). When the lump passes through the center of the condensate, its velocity is $v_{\text {lump }} \approx 9.44$, which is very close to the theoretical value Eq. (25):

$$
v_{\mathrm{th}}=\sqrt{2} \rho_{0}\left(1-\frac{3}{8} \varepsilon\right) \approx 9.4,
$$

calculated for the real lump depth $\left(\varepsilon=\varepsilon_{\text {effec }}\right)$. Note that due to the factor $3 / 8$ the lump moves faster than the plane soliton with the same depth. Besides, our calculations show that lumps are overall more stable structures than plane solitons and do not collapse during propagation even if depths are high.

Except for the background parameter, $\rho_{0}$, the dark lump is also characterized by another parameter $\varepsilon=\nu / c^{2}$, which is actually its grayness. The bigger the $\varepsilon$, the darker the lump is. The propagating velocity of the lump is described by both $\rho_{0}$ and $\varepsilon$ [Eq. (43)]. Using the parameter values given by Görlitz et al. [23] [i.e., $\omega_{z} /(2 \pi)=790 \mathrm{~Hz}, \omega_{\perp} /(2 \pi)$ $=10 \mathrm{~Hz}]$, the velocity of the lump when passing over the center of the condensate is $v_{\text {lump }}=1.94 \mathrm{~mm} \mathrm{~s}^{-1}$, larger than the velocity of the plane soliton $\left(v_{\mathrm{ps}}=1.87 \mathrm{~mm} \mathrm{~s}^{-1}\right)$ but still lower than the sound speed of the system ( $c$ $=2.33 \mathrm{~mm} \mathrm{~s}^{-1}$ ) for the same trap parameters and grayness (depth).

Near the boundary where the depth approaches its maximum value and the lump becomes black, its velocity decreases down to zero. Such configuration is unstable and we have observed nucleation of two vortices that move along the border, clockwise and counterclockwise, respectively (snapshots at $\tau=1.1$ and 1.9 in Fig. 4). For the lump we have a local decrease of condensate density and a phase offset below $\pi$ [Fig. 4(b)], while for a vortex the depth is almost $100 \%$ and the phase jump is equal to $\pi$. Then we observed a slow rotation (with angular velocity about 0.8 rad equivalent to $v_{\text {lump }} \approx 6.4$ ) of the vortices along the border of the condensate. Approaching each other at the opposite side of the condensate the vortices merge and then form a new lump soliton (Fig. 5). Accordingly, the dynamical merging of two vortices yields a lump. This follows from the stability of the dark lump in the BECs and its interaction with the boundary of the condensate.

Let us now consider collisions between dark lumps. In a previous work [18], we have shown that dark solitons in 1D BECs exhibit positive phase (or position) shifts along their paths during a head-on collision. The collisions of two dark lumps is a $2 \mathrm{D}$ problem but it can be approximated by a $1 \mathrm{D}$ case for the head-on collisions. Thus we may compare the results of two-lump collision with those obtained for twosoliton collisions in one dimension.

We initially excite two lumps [Fig. 4(a)], but moving in opposite directions along the $s_{1}$ axis (horizontal). Figure 6 shows the paths of two head-on colliding lumps [(a) and (b)] and, for reference, the pathway of the single lump (a) from a parallel experiment without the lump (b) being excited. In numerical experiments we cannot find a reliable phase shift. However, a slight mismatch between the paths of lump (a), with and without collision, can be seen. It corresponds to negative phase shift, opposite to what we have seen for $1 \mathrm{D}$ dark solitons, where as earlier mentioned the phase shift of a head-on collision was found to be positive [18]. The absence of phase shift for colliding lumps has been pointed out for a homogeneous system [40]; further details about collisions 


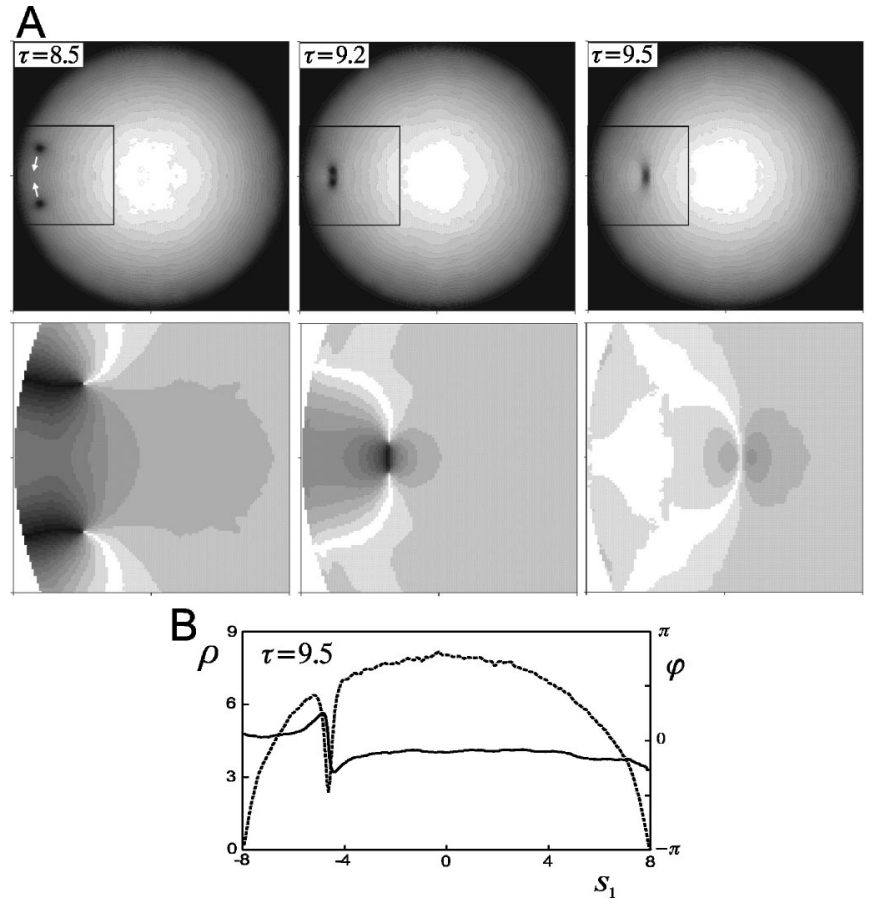

FIG. 5. Formation of a new lump by the merging of two vortices (continuation of Fig. 4). (a) Sequential snapshots of the condensate before, during, and after the merging of the vortices. The lump formed in the process propagates from the left to the right very much like the initial one (Fig. 4). (b) Section of the condensate along the horizontal axis $s_{1}$ crossing the lump for $\tau=9.5$. (solid and dashed lines correspond to the phase and the amplitude, respectively).

between algebraic and other solitons are given in, e.g., Ref. [41].

Figure 7 shows the snapshots illustrating an oblique collision of two lumps. We start with two spatially separated lumps moving from the periphery to the center of the condensate at an angle of $30^{\circ}$. At $\tau \approx 0.2$ the lumps collide, get together, and then reappear as post-collision lumps. As in head-on collisions we do not observe a significant phase shift and hence there is no deviation of trajectories after collision.

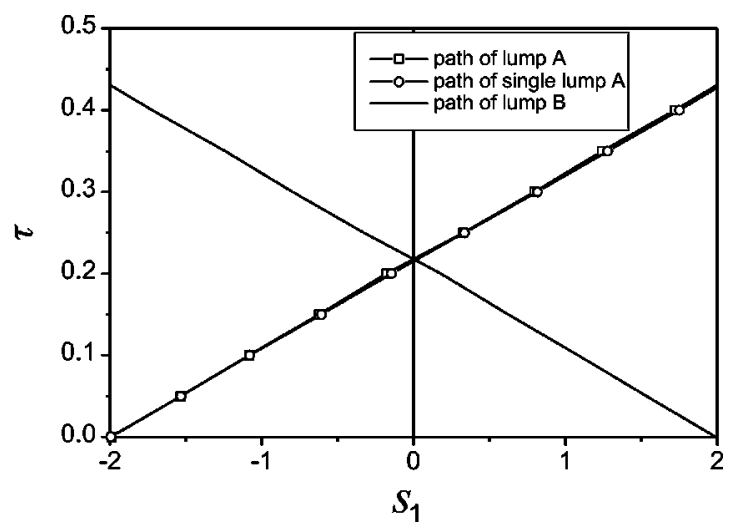

FIG. 6. Lump pathways for a head-on collision. The positions of minima of the amplitude $\rho\left(s_{1}, s_{2}\right)$ on the $s_{1}$ axis for $s_{2}=0$ are displayed.
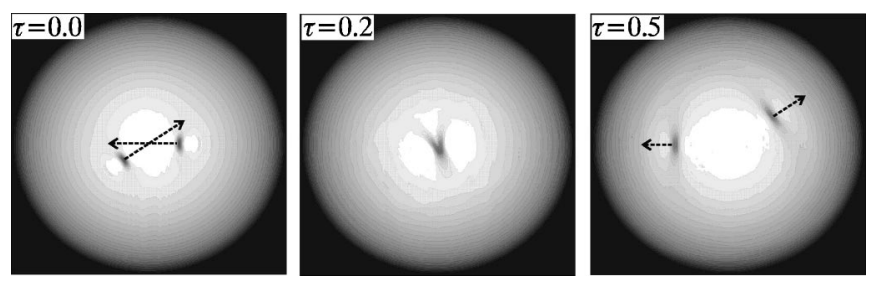

FIG. 7. Oblique collision of two lumps. Arrows show the propagation directions. Snapshots showing the amplitude, $\rho$, correspond to initial position, collision event and post-collision trajectories of the lumps.

Matsuno [41] has reported positive and negative phase shifts depending on amplitude ratios.

When both lumps (simultaneously) reach the boundary, each of them breaks down into two vortices moving clockwise and counterclockwise as in the case depicted in Fig. 4. Hence, we can say that vortices have opposite "angular momenta," while their other characteristics are identical. Then a pair of vortices moving "up" originated from different lumps collide and form a new lump that propagates to the center of the condensate. This is again similar to the process shown in Fig. 5. After some time the other pair of vortices moving "down" collide and again we get a lump. The time difference between the collisions of vortex pairs is due to the initially unequal distances between vortices (the angular difference is $2 \times 30^{\circ}$ ). Thus we get a complex sequence of events, lumps-vortices-lumps, where energy is exchanged between different lumps via vortices.

We have also tested the stability of the 1D dark lump solution corresponding to Eq. (27) when the weak trapping potential in the transverse directions is taken into account. We found that, like a dark plane soliton, a high-depth 1D dark lump is also unstable to long-wavelength transverse perturbation and decays into vortices.

\section{DISCUSSION AND SUMMARY}

We have investigated the evolution of 2D weakly nonlinear matter-wave pulses excited in a BEC with a disk-shaped trap. With repulsive atomic interaction and strong confinement in the axial direction, a Kadomtsev-Petriashvili equation with positive dispersion (KP-I) has been derived from the order-parameter equation, i.e., from the Gross-Pitaevskii equation, using a suitable asymptotic. Our results show that it is possible to excite nonlinear 2D localized excitations, i.e., dark lumps, in the system. The dynamics of dark plane solitons and 2D lumps have been thoroughly studied when including weak trapping potential in two transverse directions.

In order to explore the effect of the slowly varying trapping potential $V_{\|}(x, y)$ in the radial direction, we have made a series of numerical simulations. Numerical experiments provide evidence that a low-depth dark plane soliton can propagate in the condensate with a changing profile albeit preserving its structure until it reaches the boundary of the condensate. The interaction with the boundary is destructive and the dark plane soliton decays into a complex spatiotemporal structure including emission of sound waves and creation of vortices. Hence there is no true reflection from the 
boundary, at variance with the 1D case where oscillation of a dark soliton between the boundaries has been observed [18]. We have also shown that dark plane solitons of high depth are unstable to long-wavelength transverse perturbations, a result similar to that reported in Refs. $[14,15]$ for a 3D trap. The instability appears as a longitudinal modulation of the soliton amplitude that rapidly develops into vortices. The deeper initial soliton, the slower it moves and the faster it tears into pieces, finally decays into several vortices. An estimate of the instability wavelength gives $\lambda \approx 1$ for $l=8$.

We have shown that dark lump excitations are relatively more stable than dark plane solitons and even quite deep lumps are able to run through the condensate without decaying into vortices. When a lump approaches the boundary, it splits into two vortices that slowly move in opposite directions along the boundary at a constant distance from it. Then the vortices meet together after making a circle (or a part of a circle in the multiple lumps' case), merge and then form a new dark lump, which starts propagating through the condensate towards the center. Such process of vortices nucleation from lumps and in turn their merging into new lumps seems to be rather robust, although in computer experiments we observed non-negligible radiation of sound waves and energy losses. The geometry of the problem and properties of lumps and vortices allow vortices to form new lumps. Hence in a sense we can speak about lump-vortex similarities. We have also shown that during head-on and oblique collisions between two dark lumps, no (reliably measured) phase shift appears, at variance with the case for 1D dark solitons in the BECs where a positive phase shift was observed [18].

Recently, quasi-2D BECs have been obtained [23], considering that the energy-level spacing in the axial direction is larger than the atom-atom interaction energy and hence the projection approximation used in Eqs. (8) and (9) can be easily satisfied. To experimentally observe the $2 \mathrm{D}$ solitons and their properties here predicted, one needs a disk-shaped condensate with a large enough disk radius. The quasi-2D BEC realized by Görlitz et al. [23] can satisfy this requirement. Indeed, according to Eq. (37), the expression of the dimensionless length $l$ introduced in Eq. (36) is

$$
l=\left(\frac{4 I_{0} Q}{\pi^{3 / 2}}\right)^{1 / 4} .
$$

For the 2D disk-shaped BEC of ${ }^{23} \mathrm{Na}$ realized in Ref. [23], one has $a_{s}=2.75 \mathrm{~nm}, \quad \omega_{z} /(2 \pi)=790 \mathrm{~Hz}, \quad \omega_{\perp} /(2 \pi)$ $=10 \mathrm{~Hz}, N=2.9 \times 10^{5}$. Thus we have $a_{z}=0.74 \mu \mathrm{m}$ and $l$ $=9.1$, which corresponds to the radial size of the condensate $L_{\perp}=2\left(\omega_{z} / \omega_{\perp}\right)^{1 / 2} a_{z} l \approx 120 \mu \mathrm{m}$. In the numerical simulations for plane solitons and lumps we have required the length of the condensate to be $l=8$, which is about the same as the length of the condensate obtained in Ref. [23]. Thus the quasi-2D BEC realized in Ref. [23] can be used to test check our theoretical predictions. To excite a dark lump one may use two appropriately formed vortices that move along the boundary of the condensate and merge into a lump as shown in Fig. 5. Using the data of Ref. [23], we obtain that the period of a lump decaying into two vortices and then merging into a new lump is about $0.12 \mathrm{~s}$. The higher-order eigenmodes in the transverse directions will contribute to the soliton dynamics if the quasi-2D approximation cannot be fulfilled and the existence of thermal clouds at finite temperature will dissipate the solitons. Furthermore, an analytical approach for the soliton dynamics based on the MKP Eq. (31) remains to be formulated. These are interesting problems to be investigated in future works.

\section{ACKNOWLEDGMENTS}

The first author (G.X.H.) is grateful, for economic support and hospitality, to the Abdus Salam International Center for Theoretical Physics, Trieste, Italy, where part of this work was carried out. The second author (V.A.M.) is grateful to the Spanish Ministry of Education and Culture for economic support. This work was supported in part by the National Natural Science Foundation under Grants Nos. 19975019, 10274021, and 10205011, by the Trans-Century Program Foundation for the Talents of the Education Ministry of China, by the Shanghai Priority Academic Discipline, and by the University Key Teacher Foundation of the Education Ministry of China, and by the Spanish Ministry of Science and Technology under Grant No. PB 96-599.

\section{APPENDIX A: DERIVATION OF THE MKP EQUATION}

If a slowly varying trapping potential $V_{\|}(x, y)$ in $x$ and $y$ directions is taken into account, the asymptotic expansion presented in Sec. II B must be modified. For simplicity, we first assume $V_{\|}(x, y)=V_{\|}\left(x_{3}, y_{3}\right)$ with $x_{3}=\epsilon^{3} x$ and $y_{3}$ $=\epsilon^{3} y$. In the case of a harmonic potential, i.e., $V_{\|}$ $=\left(\omega_{\perp} / \omega_{z}\right)^{2}\left(x^{2}+y^{2}\right) / 2$, one has $V_{\|}=\Omega_{0}^{2}\left(x_{3}^{2}+y_{3}^{2}\right) / 2$ under the assumption $\omega_{\perp} / \omega_{z}=\epsilon^{3} \Omega_{0}$ with $\Omega_{0}$ a dimensionless constant of order unity. Accordingly, we make the asymptotic expansion

$$
\begin{gathered}
A=u_{0}+\epsilon^{2}\left(a^{(0)}+\epsilon^{2} a^{(1)}+\epsilon^{4} a^{(2)}+\cdots\right), \\
\varphi=\epsilon\left(\varphi^{(0)}+\epsilon^{2} \varphi^{(1)}+\epsilon^{4} \varphi^{(2)}+\cdots\right),
\end{gathered}
$$

where $u_{0}=u_{0}\left(x_{3}, y_{3}\right)$ and $a^{(j)}$ and $\varphi^{(j)}$ are the functions of the slow variables $\xi=\epsilon\left[\int^{x} c^{-1}\left(\epsilon^{3} x^{\prime}, y_{3}\right) d x^{\prime}-t\right], \quad \eta=\epsilon^{2} y$, $\tau=\epsilon^{3} t, x_{3}$, and $y_{3}$. Hence, one has the following derivative expansion:

$$
\begin{gathered}
\frac{\partial}{\partial x}=\epsilon c^{-1}\left(x_{3}, y_{3}\right) \frac{\partial}{\partial \xi}+\epsilon^{3} \frac{\partial}{\partial x_{3}}, \\
\frac{\partial}{\partial y}=\epsilon^{2} \frac{\partial}{\partial \eta}+\epsilon^{4} G\left(x_{3}, y_{3}\right) \frac{\partial}{\partial \xi}, \\
\frac{\partial}{\partial t}=-\epsilon \frac{\partial}{\partial \xi}+\epsilon^{3} \frac{\partial}{\partial \tau},
\end{gathered}
$$

where $G\left(x_{3}, y_{3}\right)=\left(\partial / \partial y_{3}\right) \int{ }^{x} c^{-1}\left(\epsilon^{3} x^{\prime}, y_{3}\right) d x^{\prime}$. Using Eqs. (A1)-(A5), Eqs. (8) and (9) with $V_{\|}(x, y)=V_{\|}\left(x_{3}, y_{3}\right)$ are transferred into

$$
\frac{\partial a^{(j)}}{\partial \xi}-\frac{1}{2} u_{0} c^{-2} \frac{\partial^{2} \varphi^{(j)}}{\partial \xi^{2}}=\alpha^{(j)}
$$




$$
\begin{aligned}
& Q^{\prime} u_{0}^{2}=\mu-\frac{1}{2}-V_{\|}\left(x_{3}, y_{3}\right), \\
& 2 Q^{\prime} u_{0}^{2} a^{(j)}-u_{0} \frac{\partial \varphi^{(j)}}{\partial \xi}=\beta^{(j)},
\end{aligned}
$$

with

$$
\begin{aligned}
& \alpha^{(0)}=0 \text {, } \\
& \alpha^{(1)}=\frac{\partial a^{(0)}}{\partial \tau}+\left(c^{-1} \frac{\partial a^{(0)}}{\partial \xi}+\frac{\partial u_{0}}{\partial x_{3}}\right) c^{-1} \frac{\partial \varphi^{(0)}}{\partial \xi} \\
& +\frac{1}{2} u_{0}\left(c^{-1} \frac{\partial^{2}}{\partial \xi \partial x_{3}}+\frac{\partial}{\partial x_{3}} c^{-1} \frac{\partial}{\partial \xi}\right) \varphi^{(0)} \\
& +\frac{1}{2} a^{(0)} c^{-2} \frac{\partial^{2} \varphi^{(0)}}{\partial \xi^{2}}+\frac{1}{2} u_{0} \frac{\partial^{2} \varphi^{(0)}}{\partial \eta^{2}}, \\
& \beta^{(0)}=0, \\
& \beta^{(1)}=\frac{1}{2} c^{-2} \frac{\partial^{2} a^{(0)}}{\partial \xi^{2}}-u_{0} \frac{\partial \varphi^{(0)}}{\partial \tau}-\frac{1}{2} u_{0} c^{-2}\left(\frac{\partial \varphi^{(0)}}{\partial \xi}\right)^{2} \\
& +a^{(0)} \frac{\partial \varphi^{(0)}}{\partial \xi}-3 Q^{\prime} u_{0}\left(a^{(0)}\right)^{2} .
\end{aligned}
$$

The explicit expressions of $\alpha^{(j)}$ and $\beta^{(j)}$ with $j \geqslant 2$ are not needed and hence are omitted here.

From Eq. (A7) one obtains $u_{0}=([\mu-1 / 2$ $\left.\left.-V_{\|}\left(x_{3}, y_{3}\right)\right] / Q^{\prime}\right)^{1 / 2}$. It is the ground configuration of the condensate in the $x$ and $y$ directions. We see that for a slowly varying trapping potential the ground state has a ThomasFermi wave-function shape. Equations (A6) and (A8) with $j=0$ have the solution

$$
\varphi^{(0)}=\frac{2 c^{2}}{u_{0}} \int^{\xi} a^{(0)}\left(\xi^{\prime}, \eta, x_{3}, y_{3}, \tau\right) d \xi^{\prime},
$$

where $a^{(0)}$ is a function yet to be determined. The solvability condition requires that

$$
c=\sqrt{Q^{\prime}} u_{0}=\left\{\left[\mu-1 / 2-V_{\|}\left(x_{3}, y_{3}\right)\right] / Q^{\prime}\right\}^{1 / 2},
$$

i.e., the system has a local sound speed, varying slowly with $x$ and $y$. The expression of the chemical potential $(\mu)$ of the system has been given in Eq. (37).

In the next order $(j=1)$, Eqs. (A6) and (A8) result in the equation for $a^{(0)}$ :

$$
\begin{gathered}
\frac{\partial}{\partial \xi}\left(\frac{\partial a^{(0)}}{\partial \tau}+\frac{3 \sqrt{Q^{\prime}}}{c} a^{(0)} \frac{\partial a^{(0)}}{\partial \xi}-\frac{1}{8 c^{2}} \frac{\partial^{3} a^{(0)}}{\partial \xi^{3}}\right)+\frac{c^{2}}{2} \frac{\partial^{2} a^{(0)}}{\partial \eta^{2}} \\
=-\frac{\partial}{\partial \xi}\left(\frac{3}{2} \frac{\partial c}{\partial x_{3}} a^{(0)}+c \frac{\partial a^{(0)}}{\partial x_{3}}\right) .
\end{gathered}
$$

It is a variable-coefficient KP equation with additional terms coming from the slowly varying trapping potential in the $(x, y)$ plane. A similar KP equation (we have called it the modified KP equation) has been obtained in weakly nonlinear water wave theory when considering a solitary wave propagating in a water channel with varying depth and width [42].

When $V_{\|}(x, y)=V_{\|}\left(x_{i}, y_{i}\right)$ with $i \geqslant 4$, a similar approach yields the local sound speed $c=\sqrt{Q^{\prime}} u_{0}=([\mu-1 / 2$ $\left.\left.-V_{\|}\left(x_{i}, y_{i}\right)\right] / Q^{\prime}\right)^{1 / 2}$. In this case the controlling equation for $a^{(0)}$ is the same as Eq. (A15) but the terms on the right-hand side disappear. Thus when $V_{\|}(x, y)=V_{\|}\left(x_{i}, y_{i}\right) \quad(i \geqslant 3)$ we have the general equation controlling $a^{(0)}$ in the form

$$
\begin{gathered}
\frac{\partial}{\partial \xi}\left(\frac{\partial a^{(0)}}{\partial \tau}+\frac{3 \sqrt{Q^{\prime}}}{c} a^{(0)} \frac{\partial a^{(0)}}{\partial \xi}-\frac{1}{8 c^{2}} \frac{\partial^{3} a^{(0)}}{\partial \xi^{3}}\right)+\frac{c^{2}}{2} \frac{\partial^{2} a^{(0)}}{\partial \eta^{2}} \\
=-\delta_{i 3} \frac{\partial}{\partial \xi}\left(\frac{3}{2} \frac{\partial c}{\partial x_{i}} a^{(0)}+c \frac{\partial a^{(0)}}{\partial x_{i}}\right),
\end{gathered}
$$

where $\delta_{i j}$ is Kronecker delta.
[1] F. Dalfovo, S. Giorgini, L.P. Pitaevskii, and S. Stringari, Rev. Mod. Phys. 71, 463 (1999), and references therein.

[2] A.J. Leggett, Rev. Mod. Phys. 73, 307 (2001).

[3] M.R. Matthews, B.P. Anderson, P.C. Haljan, D.S. Hall, C.E. Wieman, and E.A. Cornell, Phys. Rev. Lett. 83, 2498 (1999); K.W. Madison, F. Chevy, W. Wohlleben, and J. Dalibard, ibid. 84, 806 (2000); J.R. Abo-Shaeer, C. Raman, J.M. Vogels, and W. Ketterle, Science 292, 476 (2001).

[4] J. Denschlag, J.E. Simsarian, D.L. Feder, Ch.W. Clark, L.A. Collins, J. Cubizolles, L. Deng, E.W. Hagley, K. Helmerson, W.P. Reinhardt, S.L. Rolston, B.I. Schneider, and W.D. Phillips, Science 287, 97 (2000).

[5] S. Burger, K. Bongs, S. Dettmer, W. Ertmer, K. Sengstock, A. Sanpera, G.V. Shlyapnikov, and M. Lewenstein, Phys. Rev. Lett. 83, 5198 (1999).

[6] Z. Dutton, M. Budde, C. Slowe, and L.V. Hau, Science 293, 663 (2001).
[7] L. Khaykovich, F. Schreck, G. Ferrari, T. Bourdel, J. Cubizolles, L.D. Carr, Y. Castin, and C. Salomon, Science 296, 1290 (2002).

[8] K.E. Strecker, G.B. Partridge, A.G. Truscott, and R.G. Hulet, Nature (London) 417, 150 (2002).

[9] L. Deng, E.W. Hagley, J. Wen, M. Trippenbach, Y. Band, P.S. Julienne, J.E. Simsarian, K. Helmerson, S.L. Rolston, and W.D. Phillips, Nature (London) 398, 218 (1999).

[10] E.V. Goldstein and P. Meystre, Phys. Rev. A 59, 1509 (1999); 59, 3896 (1999); K. Rzazewski, M. Trippenbach, S.J. Singer, and Y.B. Band, ibid. 61, 013606 (1999); M.G. Moore and P. Meystre, Phys. Rev. Lett. 83, 5202 (1999); Y. Wu, X. Yang, C.P. Sun, X.J. Zhou, and Y.Q. Wang, Phys. Rev. A 61, 043604 (2000); J. Heurich, H. Pu, M.G. Moore, and P. Meystre, ibid. 63, 033605 (2000); M. Trippenbach, Y.B. Band, and P.S. Julienne, ibid. 62, 023608 (2000). 
[11] V.M. Perez-Garcia, H. Michinel, and H. Herrero, Phys. Rev. A 57, 3837 (1998); A.D. Jackson, G.M. Kavoulakis, and C.J. Pethick, ibid. 58, 2417 (1998); O. Zobay, S. Potting, P. Meystre, and E.M. Wright, ibid. 59, 643 (1999); A.E. Muryshev, H.B. van Linden van den Heuvell, and G.V. Shlyapnikov, ibid. 60, R2665 (1999).

[12] Th. Busch and J.R. Anglin, Phys. Rev. Lett. 84, 2298 (2000); M.D. Girardeau and E.M. Wright, ibid. 84, 5691 (2000); K.V. Kheruntsyan and P.D. Drummond, Phys. Rev. A 61, 063816 (2000); L.D. Carr, C.W. Clark, and W.P. Reinhardt, ibid. 62, 063610 (2000).

[13] A. Trombettoni and A. Smerzi, Phys. Rev. Lett. 86, 2353 (2001); P. Ohberg and L. Santos, ibid. 86, 2918 (2001); Th. Busch and J.R. Anglin, ibid. 87, 010401 (2001); L.D. Carr, J. Brand, S. Burger, and A. Sanpera, Phys. Rev. A 63, 051601 (2001); P.D. Drummond, A. Eleftheriou, K. Huang, and K.V. Kheruntsyan, ibid. 63, 053602 (2001); V.S. Filho, F.K. Abdullaev, A. Gammal, and L. Tomio, ibid. 63, 053603 (2001); P.V. Elyutin, A.V. Buryak, V.V. Gubernov, R.A. Sammut, and I.N. Towers, Phys. Rev. E 64, 016607 (2001); Biao Wu, Jie Liu, and Qian Niu, Phys. Rev. Lett. 88, 034101 (2002); V.V. Konotop and M. Salerno, Phys. Rev. A 65, 021602 (2002).

[14] B.P. Anderson, P.C. Haljan, C.A. Regal, D.L. Feder, L.A. Collins, C.W. Clark, and E.A. Cornell, Phys. Rev. Lett. 86, 2926 (2001).

[15] D.L. Feder, M.S. Pindzola, L.A. Collins, B.I. Schneider, and C.W. Clark, Phys. Rev. A 62, 053606 (2000).

[16] T. Tsurumi and M. Wadati, J. Phys. Soc. Jpn. 67, 2294 (1998).

[17] G. Huang, Chin. Phys. Lett. 18, 628 (2001).

[18] G. Huang, M.G. Velarde, and V.A. Makarov, Phys. Rev. A 64, 013617 (2001).

[19] G. Huang, J. Szeftel, and S. Zhu, Phys. Rev. A 65, 053605 (2002)

[20] M.H. Anderson, J.R. Ensher, M.R. Matthews, C.E. Wieman, and E.A. Cornell, Science 269, 198 (1995).

[21] D.S. Jin, J.R. Ensher, M.R. Matthews, C.E. Wieman, and E.A. Cornell, Phys. Rev. Lett. 77, 420 (1996).

[22] D.S. Petrov, M. Holzmann, and G.V. Shlyapnikov, Phys. Rev. Lett. 84, 2551 (2000).

[23] A. Görlitz, J.M. Vogels, A.E. Leanhardt, C. Raman, T.L. Gustavson, J.R. Abo-Shaeer, A.P. Chikkatur, S. Gupta, S. Inouye, T. Rosenband, and W. Ketterle, Phys. Rev. Lett. 87, 130402 (2001).

[24] M.J. Ablowitz and H. Segur, J. Fluid Mech. 92, 691 (1979).
[25] M.J. Ablowitz and H. Segur, Solitons and the Inverse Scattering Transform (SIAM, Philadelphia, PA, 1981), Chap. 4.

[26] A. Hasegawa and Y. Kodama, Solitons in Optical Communications (Clarendon, Oxford, England, 1995), Chap. 3.

[27] E. Zaremba, Phys. Rev. A 57, 518 (1998).

[28] H. Michinel, V.M. Pérez-García, and R. de la Fuente, Phys. Rev. A 60, 1513 (1999).

[29] S. Pötting, P. Meystre, and E.M. Wright, e-print cond-mat/0009289.

[30] D.S. Petrov, G.V. Shlyapnikov, and J.T.M. Walraven, Phys. Rev. Lett. 85, 3745 (2000).

[31] Y. Castin, in Coherent Atomic Matter Waves, edited by R. Kaiser, C. Westbrook, and F. David (EDP Sciences/Les Ulis, France, Springer-Verlag/Berlin, 2001), pp. 1-36; e-print cond-mat/0105058.

[32] L.D. Carr and Y. Castin, e-print cond-mat/0205624.

[33] $\alpha^{(j)}$ and $\beta^{(j)}$ in Eqs. (13) and (14) can be obtained from Eqs. (A9) -(A12) by setting $\partial / \partial x_{3}=\partial / \partial y_{3}=0$.

[34] E. Infeld and G. Rowlands, Nonlinear Waves, Solitons and Chaos, 2nd ed. (Cambridge University Press, Cambridge, England, 2000).

[35] M.J. Ablowitz and P.A. Clarkson, Solitons, Nonlinear Evolution Equations and Inverse Scattering (Cambridge University Press, Cambridge, England, 1991).

[36] E. Infeld, A. Senatorski, and A.A. Skorupski, Phys. Rev. E 51, 3183 (1995); D.E. Pelinovsky, Y.A. Stepanyants, and Y.S. Kivshar, ibid. 51, 5016 (1995).

[37] A.A. Nepomnyashchy, M.G. Velarde, and P. Colinet, Interfacial Phenomena and Convection (CRC-Chapman and Hall, 2002).

[38] Y.S. Kivshar and B. Luther-Davies, Phys. Rep. 298, 81 (1998).

[39] L.M. Pismen, Vortices in Nonlinear Fields. From Liquid Crystals to Superfluids. From Non-Equilibrium Patterns to Cosmic Strings (Clarendon Press, Oxford, 1999).

[40] M.J. Ablowitz and H. Segur, Solitons and Inverse Scattering Transform (SIAM, Philadelphia, 1981), Chap. 3.

[41] Y. Matsuno, J. Phys. A 12, 619 (1979); 13, 1519 (1980); D.W. Aossey, S.R. Skinner, J.L. Cooney, J.E. Williams, M.T. Gavin, D.R. Andersen, and K.E. Lonngren, Phys. Rev. A 45, 2606 (1992); Y. Matsuno, Phys. Rev. Lett. 69, 609 (1992); Y. Matsuno, Phys. Rev. E 49, 2091 (1994), and references therein.

[42] D. David, D. Levi, and P. Winternitz, Stud. Appl. Math. 76, 133 (1987) 